\title{
Evaluación del peso de conejos para carne alimentados con diferentes ensilajes
}

\section{Assessment on the weight of rabbits for meat fed on different ensilage types}

Fecha de recepción: 19 de enero de 2016

Rigoberto Villa-Ramírez ${ }^{1}$

Fecha de aceptación: 25 de mayo de 2016

Jaime Hurtado-Villegas ${ }^{2}$

\section{Resumen}

En la Granja Experimental Bengala, de la Universidad del Quindío, ubicada en el municipio de Filandia, Quindío, se evaluó la ganancia de peso en conejos tipo carne cuando son alimentados con diferentes ensilajes: pasto imperial (Axonopus scoparius), pasto imperial mezclado con botón de oro (Tithonia diversifolia (Hemsl.), pasto imperial con ramio (Boehmeria nivea) y pasto imperial con morera (Morus alba). Se valoraron cuatro tratamientos en cuatro grupos de cinco conejos cada uno. Los conejos alimentados con ensilaje de pasto imperial y botón de oro fueron los que obtuvieron el mayor peso (2389 $\mathrm{g}$, en promedio), mientras que los alimentados con solo forrajes frescos obtuvieron un peso promedio de 2021 g. Las fuentes nutricionales que mejor responden en la alimentación de conejos son, en su orden: ensilaje de botón de oro, ensilaje de morera y ensilaje de ramio.

Palabras clave: alimentación de conejos; ensilajes para conejos; forrajes; conejo Nueva Zelanda.

\begin{abstract}
At the Quindío's University experimental farm "Bengala", located in the municipality of Filandia, Quindío, the weight increase in rabbits for meat that were fed on different fodder was evaluated. The four treatments corresponded to the following ensilages: imperial grass (Axonopus scoparius), imperial grass mixed with meadow buttercup (Tithonia diversifolia (Hemsl.), imperial grass mixed with ramie (Boehmeria nivea), and imperial grass mixed with morus/mulberry (Morus alba). The evaluation was applied on four probing groups consisting of five rabbits each. The group fed on imperial grass and meadow buttercup gained the highest increase of weight (2389 $\mathrm{g}$ in average), whereas the group fed on simply fresh fodder gained a
\end{abstract}

1 M. Sc. Universidad del Quindío (Armenia, Colombia). rivilla@uniquindio.edu.co.

2 M. Sc. Universidad del Quindío (Armenia, Colombia). jhurtadov@uniquindio.edu.co. 
lower mean weight of $2021 \mathrm{~g}$. The food sources that better met the nutritional parameters of the rabbits were the following ensilages in due order: meadow buttercup, morus/mulberry, and ramie.

Keywords: dietary fiber; ensilages; New Zealand rabbits; rabbit feeding. 


\section{Introducción}

La búsqueda de alternativas de alimentación que consideren el uso de recursos disponibles en lo local constituye un elemento importante en la generación de formas de producción adecuadas para el trópico (1). El ensilaje es un método de preservación para el forraje húmedo, y su objetivo es la conservación del valor nutritivo del alimento durante el almacenamiento; este proceso permite almacenar alimento en tiempos de cosecha, conservando calidad y palatabilidad, lo cual posibilita aumentar la carga animal por hectárea y sustituir o complementar concentrados (2).

La calidad del ensilaje es afectada por la composición química de la materia que se va a ensilar, por el clima y los microorganismos empleados, entre otros factores. El ensilaje se almacena en silos que permiten mantener la condición anaerobia. Existen varios tipos de silos, y la escogencia del apropiado depende del tipo de explotación pecuaria, de los recursos económicos disponibles y de la topografía del terreno, entre otros.

El ensilaje es el producto final que se obtiene cuando se conserva un alimento mediante un proceso de fermentación anaeróbico en estado húmedo; su propósito es mantener el valor nutritivo original del alimento, con un mínimo de pérdidas de materia seca y sin que se formen productos tóxicos que puedan perjudicar las funciones productivas y la salud de los animales (3).

Otra de las características del ensilaje es la preservación de los forrajes; según Ashbell et al. (2), es una técnica conocida desde hace mucho tiempo, y es muy popular en América del Norte y en Europa. El uso del ensilaje en gran escala requiere una mínima inversión en construcciones y equipos y un plan de trabajo muy estricto y bien coordinado durante las diversas fases del proceso; además, para ensilar bajo condiciones tropicales se debe prestar especial atención a tres aspectos muy importantes: a) la madurez de la planta, la cual depende de las condiciones climáticas; en las zonas cálidas, el tiempo necesario para llegar a la madurez puede ser mucho más corto, y los cambios pueden ocurrir más rápidamente que en climas templados; en tales casos, es más difícil controlar el momento óptimo para la cosecha, lo cual es un momento crítico en los cultivos de cereales en los últimos estadios de madurez (2); b) el contenido correcto de materia seca de la planta antes del ensilado, el cual es un factor importante para el éxito de la fermentación; los imprevistos climáticos, como sequías, lluvias o altas temperaturas pueden dañar el cultivo y aumentar las pérdidas; 3) la estabilidad aeróbica: el ensilaje se puede deteriorar rápidamente; especialmente, durante la fase de distribución bajo climas cálidos y húmedos se reduce la calidad del forraje y se producen pérdidas; las temperaturas altas facilitan el desarrollo de mohos y la actividad de las levaduras durante todo el año, razón por la cual debe evitarse el ingreso de aire dentro del silo y asegurar un triturado fino, buena compactación y sellado; al abrir el silo y retirar ensilaje se debe cuidar que la extracción deje una pared muy plana con el mínimo de superficie expuesta al aire. La estabilidad aeróbica debería ser una prueba rutinaria en zonas calientes (4).

Con este sistema se obtiene un alimento de aceptable a bueno en cuanto a calidad nutricional, empleando una mezcla de alimentos ricos en carbohidratos fermentables junto con substrato proteico no fermentable (5).

El conejo es una especie fundamentalmente crepuscular y nocturna; pertenece al orden Lagomorpha, familia Lepóridae, género Oryctolagus, especie Oryctolagus cuniculus L. (6). Según Velázquez (7), el conejo es un animal herbívoro, vivaz, su alimentación es variada e ingiere la comida a gran velocidad; es resistente a las condiciones ambientales adversas, pues sus mecanismos digestivos y su cobertura cutánea le permiten hacer frente al frío, a la falta de alimentos y a otras condiciones adversas. Su ansiedad permanente y su frecuente inquietud están en relación directa con la densidad poblacional en que se encuentre, causando graves repercusiones sobre su fisiología, como la inhibición de los procesos digestivos, la ovulación provocada en hembras o la esterilidad en los machos. Por lo 
anterior, se recomienda la explotación de los conejos en jaulas individuales (8).

Los conejos presentan algunas peculiaridades anatómicas (como el desarrollo de sus pabellones auriculares) y fisiológicas (por ejemplo, la cecotrofia y la capacidad de las hembras para la ovulación estimulada). Por medio del proceso de la cecotrofia, el conejo obtiene un suplemento de vitaminas de complejo $B$, proteínas de calidad que representan partes de sus necesidades nutritivas; el conejo aprovecha un gran porcentaje de alimentos fibrosos, cuya digestión sería factible de no existir este singular reciclaje (9).

El conejo, por su eficiencia reproductiva, su rusticidad y su fácil alimentación y manejo, se ha convertido en una seria e importante alternativa para el pequeño y mediano productor, ya que cada día adquiere más importancia como productor alimentario, pues su índice de conversión de alimentos es muy alto; solamente se necesita de 2,5 a $3,5 \mathrm{~kg}$ de alimento para producir un kilogramo de carne (10).

La Asociación Americana de Cunicultura reconoce poco más de 45 razas de conejos; pero no todas se producen de manera comercial, sola unas cuantas han alcanzado un desarrollo económico interesante, de ahí que, por aspectos prácticos, se seleccionaron aquellas razas más comunes y de mayor uso en sistemas de explotación comercial a nivel tropical para ser estudiadas (9).

Entre los criterios para clasificar las razas de conejos se mencionan los siguientes: razas productoras de carne, razas productoras de piel o pelo, razas pequeñas empleadas como mascotas y razas de doble propósito (8).

La raza neozelandés es considerada productora de carne; procede del cruzamiento de Blanco Americano y Angora; es de ojos rojos, brillantes y expresivos; su cuerpo es de longitud media, compacto, redondeado y con buen desarrollo muscular; su longitud aproximada es de $47 \mathrm{~cm}$ para machos y de 49,5 cm para hembras (midiendo de la punta de la cola a la punta del hocico). Existen diferentes variedades de la raza nueva zelanda, como son: negra, roja y blanca (6). Los conejos de esta raza tienen grandes masas musculares, el lomo y la grupa son rellenas y redondeadas; el peso ideal del macho adulto es de $4,5 \mathrm{~kg}$, y el de la hembra, de $5 \mathrm{~kg}$. Esta raza se caracteriza por su habilidad materna, docilidad, precocidad y ser de buena calidad peletera (calidad de piel para manufactura de artículos como bolsos, carteras y guantes entre otros). Además, presenta un buen rendimiento en canal, adaptado a diferentes climas, y son muy prolíficos (9).

Bajo este contexto, la presente investigación buscó evaluar la condición nutricional de diferentes ensilajes y medir los aumentos de peso en conejos para carne desde el destete hasta el momento de la comercialización, ya que en el Quindío están tomado auge las explotaciones cunículas, donde los animales son alimentados con forraje verde y concentrado comercial.

\section{Materiales y métodos}

\section{A. Localización.}

La investigación se realizó en el municipio de Filandia, Quindío, vereda Cruces, a los $4^{\circ} 40^{\prime}$ 48.7" de latitud norte y $75^{\circ} 39^{\prime} 48.5^{\prime \prime}$ de longitud oeste, en la "Granja Experimental Bengala", de propiedad de la Universidad del Quindío, la cual tiene una altitud de $2050 \mathrm{~m}$, una temperatura promedio de $18{ }^{\circ} \mathrm{C}$ y un promedio anual de lluvia de $2800 \mathrm{~mm}$.

\section{B. Diseño de tratamientos.}

Para elaborar los ensilajes se siguió el método propuesto por Barbado (10) y Betancourt et al. (11). Todos los forrajes para ensilar se cosecharon antes de iniciar su fase de floración (70-120 días); posteriormente, se sometieron a una deshidratación en campo y, una vez obtenido un 30 o $40 \%$ de pérdida de gua, se realizó el picado del material, entre 3 y $5 \mathrm{~cm}$, aproximadamente; luego, se procedió a mezclar el material forrajero fragmentado, adicionándole melaza en una proporción de $1 \mathrm{~kg}$ por cada $50 \mathrm{~kg}$ de forraje por ensilar. Las mezclas se empacaron en bolsas negras en forma compacta, para sacar todo el aire del silo, 
y se mantuvieron bajo condiciones anaeróbicas, de tal forma que se favorezca una buena fermentación; las bolsas de silos se almacenaron en estivas en un lugar limpio, seco y fresco. Los cuatro ensilajes fueron:

- Tratamiento 1. Pasto Imperial (Axonopus scoparius (Flüggé) Kuhlm): 100\%.

- Tratamiento 2. Pasto Imperial y Botón de Oro (Tithonia diversifolia (Hemsl.): $70 \%$ y $30 \%$.

- Tratamiento 3. Pasto Imperial y Morera (Morus alba L.): $70 \%$ y $30 \%$.

- Tratamiento 4. Pasto Imperial y Ramio (Boehmeria nivea L. Gaudich): 70\% y 30\%.

\section{Obtención de animales.}

Se preñaron e iniciaron las gestaciones de las conejas de la raza Nueva Zelanda, para la obtención de los gazapos necesarios para adelantar el trabajo en campo; pasados 30 días de edad fueron destetados y se iniciaron los tratamientos de alimentación mencionados.

Se conformaron de manera aleatoria cuatro grupos de cinco conejos de camadas homogéneas (mismo sexo, misma edad e igual cruce racial), los cuales se ubicaron en jaulas tipo batería debidamente acondicionadas; para que existiera homogeneidad entre los tratamientos se tomaron los pesos iniciales de cada uno de los animales y se conformaron los diferentes grupos según el peso promedio: G1: $144 \mathrm{~g}$ en promedio (719g peso total de los 5 individuos), al cual se le aplicó el T1; G2: 139g (693g peso total), al cual se le aplicó el T2; G3: 145g (723g peso total), al cual se le aplicó el T3, y G4: 144g (719g peso total), al cual se le aplicó el T4. Los animales se sometieron a una semana de adaptación; durante los primeros cuatro días se les suministró alimento balanceado más el ensilaje, ambos a libre consumo, y en los últimos tres días fueron alimentados exclusivamente con el tratamiento correspondiente. Los conejos fueron alimentaron durante 90 días hasta alcanzar el peso de sacrifico.

\section{Variables a evaluadas.}

Se evaluaron los siguientes aspectos del ensilaje: características físicas y organolépticas de color, olor, textura, sabor (palatabilidad) y consistencia, para cada uno de los silos elaborados (27).

Se realizó un análisis bromatológico (Tabla 2), en el Laboratorio de Poscosecha de la Universidad del Quindío. Los conejos fueron pesados individualmente cada semana, en cada tratamiento, con el fin de terminar la ganancia de peso.

\section{E. Diseño experimental.}

Se utilizó un diseño experimental completamente al azar (12), con cuatro tratamientos (fuentes nutricionales) y cinco repeticiones por tratamiento, siendo la unidad experimental un conejo. Se registraron semanalmente los datos de peso de cada conejo a la misma hora y por un lapso aproximado de tres meses; se evaluó el aumento de peso (medido en gramos), dado por el promedio de los conejos alimentados con las cuatro fuentes nutricionales.

Para evaluar las variables descritas se procedió a realizar el análisis estadístico ANDEVA, y se utilizó la prueba de comparación de medias de Tukey para la variable ganancia de peso semanal y total; se empleó el sistema estadístico Statgraphics Centurión XVI.

\section{Resultados y discusión}

Se obtuvieron ensilajes maduros que físicamente mostraron color café claro y olor agradable; la textura, o consistencia, de los ensilados se consideró buena cuando el material conservó todos sus contornos definidos; la determinación de la humedad en el material fermentado se consideró excelente, ya que humedecía las manos al ser comprimido dentro del puño y se mantenía suelto después de retirada la presión; la palatabilidad se evacuó teniendo en cuenta la aceptación o rechazo de los conejos a la hora de suministrarles los ensilajes (10).

Según las características bromatológicas (Tabla I) de los diferentes ensilajes se pudo diferenciar el componente proteico entre los silos preparados a partir de asocios de forrajes. 
Tabla I. Características bromatológicas para ensilajes elaborados a partir de pasto imperial con botón de oro, ramio y morera.

\begin{tabular}{lccccc}
\hline \multicolumn{1}{c}{ ANÁLISIS } & T 1 & T 2 & T 3 & T 4 & Método \\
\hline Humedad (\%) & 54.02 & 76.44 & 69.01 & 71.21 & AOAC 969.38 B \\
Cenizas (\%) & 10.03 & 10.44 & 8.02 & 12.56 & AOAC 942.05 \\
Grasa (\%) & 1.07 & 0.73 & 1.02 & 0.82 & NTC 668 \\
Proteína* (\%) & 4.90 & 8.28 & 6.66 & 8.95 & AOAC 978.04 \\
\hline
\end{tabular}

AOAC: Internacional "Asociación de las Comunidades Analíticas" NTC: Norma Técnica Colombiana

*El porcentaje de proteína es en base seca; el factor utilizado es de 6,25, correspondiente a todos los otros alimentos.

El porcentaje de humedad en los ensilajes oscila entre el $54 \%$ y el $76 \%$. Barbado (10) indica que los ensilajes deben portar gran contenido de humedad; así, todos los ensilajes se encuentran en un rango óptimo de porcentaje de humedad, según De La Raza (13).

El contenido de cenizas para los distintos tratamientos fluctuó entre el $8,02 \%$ y el 12,56 $\%$; contenidos mayores a $12 \%$ de ceniza son asociados a contaminación con suelo durante la cosecha o la elaboración del ensilaje (14), lo que favorece la presencia de fermentaciones secundarias y reducción del consumo, situación que no sucede en el presente trabajo. Según Guzmán et al. (15), el porcentaje de cenizas que presentan investigaciones en forrajes ensilados está entre el $5 \%$ y el $10 \%$. Para De La Raza (3), las cenizas deseables en un ensilaje se situarían en un máximo de $12 \%$ y, como se puede observar, el silo de pasto imperial y ramio superó dicho porcentaje. En la conservación por ensilajes, se trata de inhibir el crecimiento de microorganismos degradadores de la materia seca (16).

El porcentaje de grasa en cualquier ensilaje está descrito por los ácidos grasos volátiles, que son producto de los ácidos orgánicos, como el ácido láctico y el acético, entre otros. Según esta investigación, la mayor concentración de grasas la tiene el pasto imperial $(1,07 \%)$. El valor mínimo de ácidos grasos que requiere un ensilaje agradable y catalogado como de buenas características es de
$1,5 \%$ a $2 \%$, mientras que un $6 \%$ se estima como muy malo (17).

Barrionuevo (2011) menciona que cuando los conejos son alimentados con contenidos proteicos menores del $16 \%$ y contenido de fibra cruda entre el $12 \%$ y el $15 \%$, el conejo muestra normalidad digestiva y un crecimiento bajo; en cambio, cuando la proteína bruta es del $16-18 \%$ y el mismo contenido de fibra hay normalidad digestiva, pero el crecimiento es normal

La calidad final del ensilado depende tanto de las materias primas como de la aplicación adecuada de la técnica. Entre los factores de la materia prima destacan la altura de corte, el nivel de humedad, el tamaño de las partículas, la porosidad de la masa forrajera (18), la resistencia a la compactación (19) y la calidad fermentativa, determinada por la concentración de ácidos orgánicos, nitrógeno amoniacal y $\mathrm{pH}$ (7). En general, los forrajes con una concentración de 6-8\% de carbohidratos solubles y un contenido de materia seca del $32-35 \%$, con bajo poder tampón, constituyen una adecuada materia prima para el ensilaje (20).

Por medio del ensilaje se puede almacenar y reservar el alimento para su uso posterior con pérdidas mínimas de calidad nutricional (16), (21) y (18).

En el caso de las zonas tropicales y subtropicales, el ensilado de cultivos forrajeros o subproductos industriales podría ser una contribución importante 
para optimizar el funcionamiento de los sistemas de producción animal (16); sin embargo, debe tenerse en cuenta la composición química del material que se va a ensilar, ya que juega un papel importante en la determinación de su potencial; se conservan de mejor manera forrajes con altos niveles de azúcares fermentables, bajo nivel de proteína, baja capacidad de amortiguación del pH y un mínimo contenido de materia seca (MS) (22). Estas condiciones se presentan difícilmente con los materiales disponibles en las zonas tropicales (23).

En la Figura 1, se puede observar el peso inicial de cada grupo (después del destete), el peso final y el aumento en peso vivo en cada uno de los grupos alimentados con los diferentes ensilajes a los 90 días.

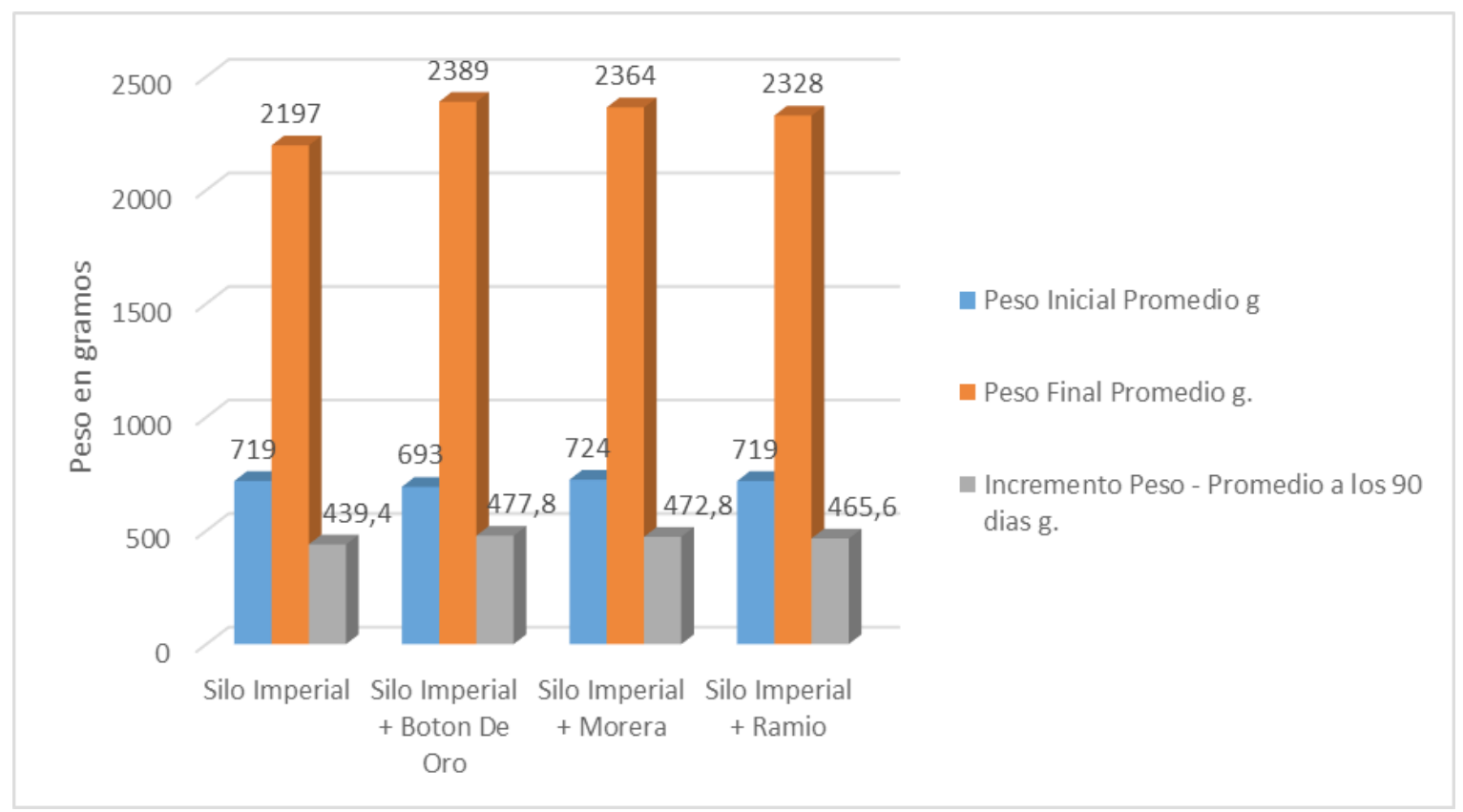

Fig. 1. Peso promedio de conejos alimentados con diferentes ensilajes a los 90 días.

El grupo de conejos alimentados con el T2 (Pasto Imperial + Boton de Oro) mostró el mejor desempeño en cuanto al aumento de peso $(477,8 \mathrm{~g})$. De este resultado se desprenden dos valoraciones: en primer lugar, que los ensilajes se convierten en una alternativa real para la alimentación de conejos destinados a la producción de carne, y cuando estos ensilajes se elaboran a partir de asocios de forrajes (imperial con botón de oro, morera o ramio) se fortalecen los valores nutricionales de estos, lo que redunda en un comportamiento más adecuado en la intención de producir carne en la empresa cunícola.

El análisis estadístico no presenta diferencias significativas $(p<0.05)$ entre los tratamientos T1 y T2 y T1 y T3, mientras que entre los tratamientos
T1 y T4 sí existen diferencias significativas, lo cual confirma que el grupo alimentado con ensilaje de pasto Imperial + Botón de Oro fue el de mayor aumento en peso $(477,8 \mathrm{~g})$; el botón de oro ha sido reconocido entre los productores como una planta con un importante valor nutricional, principalmente por su capacidad para la acumulación de nitrógeno $(24,25)$ y por el nivel de fibra bruta, que es del $31,6 \%$ a los 60 días de edad (26), características que dejan al botón de oro en condiciones nutricionales similares a las de otras plantas arbustivas destinadas a la producción forrajera.

Algunos estudios han demostrado que el conejo tiene la posibilidad de utilizar leguminosas y gramíneas forrajeras como parte de la dieta sin 
desmejorar significativamente la ganancia de peso (28), en tal sentido, se demuestra que las plantas forrajeras tienen gran importancia para alimentación animal en el trópico.

\section{Conclusiones}

Los conejos alimentados con ensilaje Pasto Imperial + Botón de Oro obtienen mayor ganancia de peso que los animales alimentados con los demás ensilajes.

Cuando los ensilajes son elaborados con asocio de gramíneas y forrajes se fortalece la ración alimenticia, lo que redunda en un mejor desempeño por parte del animal, en este caso una ganancia de peso.

Para la alimentación cunícola es importante el suministro de los nutrientes requeridos en cantidades adecuadas, los cuales se pueden reemplazar ofreciendo ensilajes como dieta total o complementada con alimento balanceado comercial, ya que se puede balancear de tal manera que cumpla con los requerimientos nutricionales del animal.

\section{Agradecimientos}

A la Vicerrectoría de Investigaciones de la Universidad del Quindío, por su financiación.

\section{Referencias}

(1) Nieves D, Terán O, Silva L, González C. Digestibilidad in vivo de nutrientes en dietas en forma de harina con niveles crecientes de Leucaena leucocephala para conejos de engorde. Rev. Científ, 2002; 12(2): 408-411.

(2) Ashbell G, Weinberg ZG, Bolsen KK, Hen Y, Azrieli A. The silage characteristics of two varieties of forage sorghum mixed in different proportions at two stages of maturity. African J. Range Forage Sci., 1999; 15:69-72.

(3) García G. Ensilado de Forrajes. Madrid, España: Publicaciones de Extensión Agraria, 1991.
(4) Filippi R. Conceptos básicos en la elaboración de ensilajes. Chile: Universidad de la Frontera, 2011.

(5) Aguilera A, Pérez F, Grande D, De la Cruz I, Juárez J. Digestibility and fermentative characteristics of mango, lemon and corn stover silages with or without addition of molasses and urea. Small Ruminant Research, 2007; 26: 87-91. DOI: http://dx.doi.org/10.1016/S09214488(96)01001-2.

(6) Echeverry J. Explotación y manejo de conejo doméstico. Politécnico Colombiano Escuelas de Ciencias Agrarias. Politécnico Colombiano Jaime Isaza Cadavid, 2004.

(7) Velázquez R. Evaluación genética del comportamiento productivo y reproductivo en núcleos de conejos de las razas Nueva Zelanda y Chinchilla, Revista CORPOICA Ciencia y Tecnología Agropecuaria, 2007; 8(1): 69-74. DOI: http://dx.doi.org/10.21930/rcta.vol8_ num1_art:86.

(8) Roca J, Martínez S, Orengo J, Parrilla I, Vázquez JM, Martinez EA. Influence of constant long days on ejaculate parameters rabbits reared under natural environment conditions of Mediterranean area. Livestock Production Science, 2005; 94: 169-177. DOI: http://dx. doi.org/10.1016/j.livprodsci.2004.10.011.

(9) González RP, Caravaca-Rodríguez FP. Producción de conejos de aptitud cárnica. Sistemas ganaderos en el siglo Xxı. España, 2007.

(10) Barbado JL. Cría de conejos, su empresa de cunicultura. Argentina: Albatros, 2006.

(10) Betancourt M, González I, Martínez de Acurero M. Evaluación de la calidad de los ensilajes. Revista Digital Centro Nacional de Investigaciones Agropecuarias de Venezuela, 2005; 8: $1-5$.

(11) Cañete MV, Sacha JL. Ensilado de forrajes y su empleo en la alimentación de rumiantes. Madrid: Mundi-Prensa, 1998. 
(12) Badii MH, Castillo J, Barragán JN, Flores AE. Análisis discriminante. En: M.H. Badii y J. Castillo (eds.): Técnicas Cuantitativas en la Investigación. Monterrey: UANL, 2007.

(13) De la Raza B. El ensilado en zonas húmedas y sus indicadores de calidad. IV Jornadas de Alimentación Animal. Laboratorio de Mouriscade. Lalín, pp. 1-20.

(14) Chaverra G. Bernal E. Ensilaje en la alimentación de ganado vacuno. Bogotá: IICA, Tercer Mundo Editores, 2000.

(15) Guzmán O, Lemus C, Martínez S, Bonilla J, Plasencia A, Ly J. Chemical characteristics of silages of mango (Mangifera indica L.) by products for animal feeding. Cuban J. Agric. Sci., 2012; 46(4): 369-374.

(16) Garcés M, Suárez E, Serna J, Ruíz S. Evaluación de la calidad bromatológica del ensilaje de pasto kikuyo y maní forrajero. Revista LasaIlista de Investigación, 2006; 3(2): 34-37.

(17) Garcés AM, Berrío L, Ruíz S, Serna JG, Builes AF. Ensilaje como fuente de alimentación para el ganado. Rev. Lasallista Investigación, 2011; 1(1): 66-71.

(18) Reyes N, Mendieta B, Fariñas T, Mena M, Cardona J, Pezo D. Elaboración y utilización de ensilajes en la alimentación del ganado bovino. Managua, Nicaragua: CATIE, 2009.

(19) Jobim C, Nussio L, Reis R, Schmidt P. Avanços metodológicos naavaliaçao da qualidade da forragem canservada. Revista Brasileña de Zootecnia, 2007; 36 (suplemento especial), 101-119.

(20) Villa AF, Meléndez AP, Carulla JE, Pabón ML, Cárdenas EA. Estudio microbiológico y calidad nutricional del ensilaje de maíz en dos ecoregiones de Colombia. Revista Colombiana Ciencias Pecuarias, 2010; 23(1): 65-77.

(21) Qamar M. Effect of molasses and corn as silage additives on the characteristics of Mott Dwarf Elephant grass silage at different fermentation periods. Pak. Vet. J., 2009; 29: 19-23.
(22) Nisa M, Shahzad MA, Sarwar M, Tauqir NA. Influence of additives and fermentation periods on silage characteristics, chemical composition, and in situ digestion kinetics of Jambo silage and its fodder in Nili buffalo bulls. Turk. J. Vet. Anim. Sci., 2008; 32(2): 67-72.

(23) Bergamaschine AF, Passipiéri $M$, Veriano WV, Isepon OJ, Almeida-Correa LD. Qualidade e valor nutritivo de silagens de capim-marandu (B. brizantha cv. Marandu) produzidas com aditivos ou forragem emurchecida. R. Bras Zootec., 2006; 35(4): 14541462. DOI: http://dx.doi.org/10.1590/S151635982006000500027.

(24) Medina M, García D, González E, Cova L, Morantinos $\mathrm{P}$. Variables morfo-estructurales y de calidad de la biomasa de Tithonia diversifolia en la etapa inicial de crecimiento. Zootecnia Trop., 2009; 27: 121-134.

(25) Verdecia D, Ramírez J, Leonard I, Álvarez Y, Bazán Y, Bodas R, Andrés S, Álvarez J, Giráldez F, López S. Calidad de la Tithonia diversifolia en una zona del Valle del Cauto. REDVET. En: http://www.veterinaria.org/revistas/ redvet/n050511/051113.pdf.

(26) Roa M, Castillo C, Téllez E. Influencia del tiempo de maduración en la calidad de ensilajes con forrajes arbóreos. Sistemas de Producción Agroecológicos, 2010; 1(1): 63-73.

(27) Betancourt M, González I, Martínez M. Evaluación de la calidad de los forrajes. Maracaibo, 2006.

(28) Triguero RO, Villalta P. Evaluación del uso de follajes deshidratado de morera (Morus alba) en la alimentación de cerdos de la raza landrece en la etapa de engorde. San SV. 1997; 150-155. 